\title{
Neoplasia inusual escamosa de la superficie ocular y fibroxantoma atípico en córnea y conjuntiva: reporte de caso
}

\section{A rare ocular surface neoplasia and atypical fibroxanthoma in cornea and conjunctiva: a case report}

\author{
Yendry Monge-Morera ${ }^{1 *}$, Lindsay Chacón ${ }^{1}$, Mario Barrantes-Domínguez ${ }^{1}$, Greivin Rodríguez-Rojas ${ }^{2}$ y \\ Erick Hernández-Bogantes ${ }^{1,3}$ \\ ${ }^{1}$ Departamento de Oftalmología, Hospital San Rafael de Alajuela, Caja Costarricense de Seguro Social (CCSS), Alajuela; ${ }^{2}$ Departamento de Patología, \\ Hospital Calderón Guardia, Caja Costarricense de Seguro Social (CCSS), San José; ${ }^{3}$ Departamento Oftalmología, Centro Ocular, Heredia. Costa Rica
}

\section{Resumen}

Se presenta el caso de un varón de 72 años que consultó por sensación de cuerpo extraño en el ojo derecho. Durante la revisión se encuentra una lesión polipoide de $12 \times 10 \times 10 \mathrm{~mm}$, vascularizada, bien circunscrita, localizada en la conjuntiva bulbar y el limbo nasal. Los hallazgos histopatológicos revelan una neoplasia intraepitelial conjuntival T2 y un fibroxantoma atípico tanto en la córnea limbal como en la conjuntiva bulbar. Se realizó una biopsia escisional con márgenes amplios, crioterapia y colocación de membrana amniótica criopreservada, con posterior aplicación de mitomicina C al 0.04\%. Después de 4 meses de seguimiento no hay evidencia de recurrencia. El fibroxantoma atípico en la superficie ocular es un hallazgo poco común. La sospecha clínica, el diagnóstico temprano y el manejo adecuado son importantes para un buen pronóstico ocular. Aunque el fibroxantoma atípico es típicamente benigno, puede haber casos de recurrencia y metástasis sistémica.

Palabras clave: Fibroxantoma atípico. Tumor conjuntival. Neoplasia de la superficie ocular. Inmunohistoquímica. Tumor corneal.

\begin{abstract}
We present the case of a 72-year-old male who consulted for a foreign body sensation in his right eye. During examination, a well-circumscribed, vascualrized $12 \times 10 \times 10 \mathrm{~mm}$ polypoid lesion was found in the bulbar conjunctiva and the nasal limbus. Histopathological findings revealed a T2 conjunctival intraepithelial neoplasia and an atypical fibroxanthoma in both the limbal cornea and the bulbar conjunctiva. An excisional biopsy with wide margins, cryotherapy and placement of a cryopreserved amniotic membrane was performed, with subsequent application of mitomycin C $0.04 \%$. After 4 months of follow-up there was no evidence of recurrence. Atypical fibroxanthoma on the ocular surface is a rare finding. Clinical suspicion, early diagnosis, and proper management are important for a good ocular prognosis. Although atypical fibroxanthoma is typically benign, there may be cases of recurrence and systemic metastasis.
\end{abstract}

Key words: Atypical fibroxanthoma. Conjunctival tumor. Ocular surface neoplasia. Immunohistochemistry. Corneal tumor.

Correspondencia:

*Yendry Monge-Morera

Radial Francisco J. Orlich

Fecha de recepción: 24-02-2021

C.P. 20101, Provincia de Alajuela, Alajuela, Costa Rica

E-mail: damongemorera@gmail.com

0187-4519/○ 2021 Sociedad Mexicana de Oftalmología. Publicado por Permanyer. Este es un artículo open access bajo la licencia CC BY-NC-ND (http://creativecommons.org/licenses/by-nc-nd/4.0/).
Disponible en internet: 25-10-2021

Rev Mex Oftalmol. (ahead of print)

www.rmo.com.mx 


\section{Introducción}

El fibroxantoma atípico (FXA) es un tumor raro de la dermis, pleomórfico, indoloro, que se presenta sobre todo en zonas con exposición solar directa. Usualmente se presenta en hombres entre la quinta y la séptima décadas de la vida ${ }^{1,2}$.

Entre sus factores de riesgo se encuentran la exposición a rayos ultravioleta, el trauma y la cicatrización previa, la historia de irradiación, el tratamiento inmunosupresor y el antecedente de algún otro tipo de cáncer de piel².

El FXA pertenece al grupo de neoplasias atípicas de tumores histiocíticos, y por lo tanto el diagnóstico definitivo se basa en la evaluación histopatológica junto con estudios de inmunohistoquímica.

Reportamos los hallazgos histopatológicos y clínicos de un caso inusual de neoplasia intraepitelial (NIC) y FXA tanto en la córnea como en la conjuntiva.

\section{Presentación del caso}

Varón de 72 años de edad con antecedentes de diabetes mellitus tipo 2, hipertensión arterial primaria y cáncer de próstata tratado con flutamida oral. Consultó por sensación de cuerpo extraño y disminución de visión en el ojo derecho de 8 meses de evolución, con exacerbación de los síntomas en las últimas 4 semanas.

Al momento de la atención presentaba una agudeza visual de 20/160 en el ojo derecho y de 20/30 en el ojo izquierdo. Con la lámpara de hendidura en el ojo derecho se observó una lesión polipoide de $12 \times 10 \times$ $10 \mathrm{~mm}$, vascularizada, de color rosa, circunscrita, localizada en la conjuntiva bulbar nasal y en el limbo, con afectación de este y de la córnea adyacente. La tinción con rosa de Bengala fue positiva. Al contacto con la lesión, no se detecta adherida a la esclera subyacente (Fig. 1 A). Inicialmente se planteó el diagnóstico clínico de una NIC.

Debido al tamaño y el rápido crecimiento del tumor, se decidió realizar de manera temprana una resección quirúrgica con la técnica no-touch, descrita por Shields y Shields ${ }^{3}$. Durante el intraoperatorio se aplicó alcohol absoluto diluido al $30 \%$ sobre el epitelio corneal afectado y se eliminó con bisturí.

Posteriormente se realizó una incisión conjuntival dejando $4 \mathrm{~mm}$ de margen libre y eliminando la totalidad de la masa, la cual fue enviada de inmediato para su evaluación histopatológica. Finalmente, se aplicó

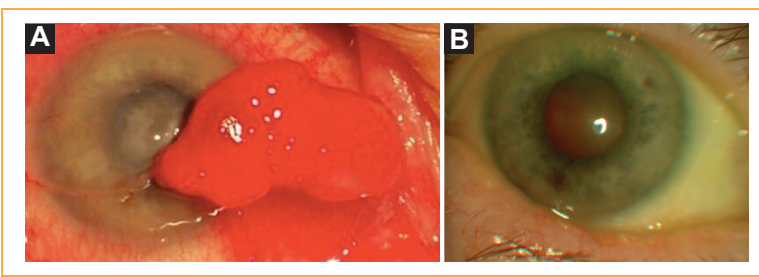

Figura 1. Fotos con lámpara de hendidura, ojo derecho. A: masa circunscrita localizada, en la conjuntiva bulbar y el limbo nasal, con involucro de la córnea periférica. B: evolución 3 meses después de la escisión y la aplicación de mitomicina C al $0.04 \%$ tópica.

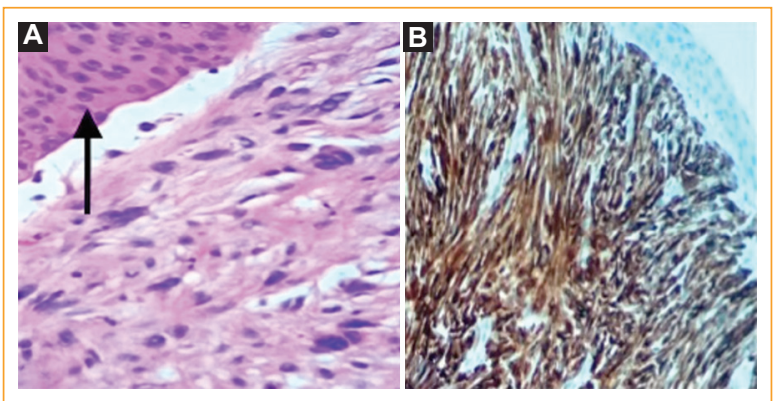

Figura 2. Corte histológico de la conjuntiva. A: displasia en el epitelio conjuntival (flecha). En el estroma se identificaron proliferación de células pleomórficas atípicas, mitosis atípica y células multinucleadas (hematoxilina-eosina, $\times 125$ ). B: inmunotinción de CD10 que muestra una importante expresión de células fusiformes, pleomórficas y atípicas.

crioterapia en la conjuntiva bulbar adyacente y se colocó membrana amniótica criopreservada con Vicryl 9-0. Posterior a la cirugía se le aplicó mitomicina $\mathrm{C}$ al $0.04 \%$ tópica cuatro veces al día durante 6 semanas. A los 4 meses de posoperatorio, la visión del ojo derecho mejoró a 20/60, sin hallazgos evidentes de recurrencia (Fig. $1 \mathrm{~B}$ ).

Histológicamente se identificó una NIC T2 (Fig. 2 A). En la sustancia propia conjuntival se observaron células organizadas en fascículos, algunas células claras en el citoplasma y otras gigantes multinucleadas, que mostraban pleomorfismo e hipercromasia nuclear y figuras de mitosis. La reacción de inmunohistoquímica para CD10 resultó intensamente positiva en la membrana de las células neoplásicas (Fig. 2 B).

Las reacciones de inmunohistoquímica para p63, antígeno de membrana epitelial, desmina, HMB45, S100, CD31 y pancitoqueratina resultaron negativas. 
Tabla 1. Características de los pacientes con fibroxantoma atípico ocular

\begin{tabular}{|c|c|c|c|c|c|c|c|}
\hline Autores & Sexo & Antecedentes & $\begin{array}{l}\text { Edad } \\
\text { (años) }\end{array}$ & Localización & Resolución & Recidiva & Lesión mixta \\
\hline Shieh, et al. ${ }^{7}$ & Femenino & Ninguno & 37 & Limbo nasal & Quirúrgica & No & No \\
\hline Shao, et al. ${ }^{4}$ & Masculino & Xeroderma pigmentoso & 5 & Limbo lateral & Quirúrgica & Sí & $\begin{array}{l}\text { Carcinoma } \\
\text { escamoso }\end{array}$ \\
\hline Kuiper, et al. ${ }^{2}$ & Masculino & $\begin{array}{l}\text { Hipertensión, } \\
\text { hipotiroidismo, } \\
\text { hiperlipidemia }\end{array}$ & 71 & Limbo nasal & Quirúrgica & No & No \\
\hline Mochizuki, et al. ${ }^{9}$ & Femenino & $\begin{array}{l}\text { Demencia, insuficiencia } \\
\text { cardiaca, evisceración }\end{array}$ & 94 & $\begin{array}{l}\text { Conjuntival, } \\
\text { palpebral } \\
\text { inferior }\end{array}$ & Quirúrgica & No & No \\
\hline Engelbrecht, et al. ${ }^{1}$ & Masculino & Trauma ocular & 59 & $\begin{array}{l}\text { Córnea/ } \\
\text { limbo }\end{array}$ & Quirúrgica & No & $\begin{array}{l}\text { Neoplasia } \\
\text { intraepitelial } \\
\text { escamosa }\end{array}$ \\
\hline Bubshair, et al. & Masculino & Xeroderma pigmentoso & 17 & Córnea & Quirúrgica & No & $\begin{array}{l}\text { Displasia } \\
\text { moderada }\end{array}$ \\
\hline Ullrich, et al. ${ }^{5}$ & Masculino & Pseudofaquia & 81 & Limbo & Quirúrgica & No & No \\
\hline
\end{tabular}

\section{Discusión}

El FXA supone el $0.2 \%$ de todos los tumores de piel. La mayoría de los casos ocurren en la cabeza, la cara y el cuello. Usualmente, el FXA se comporta como un tumor benigno, con una recurrencia de un $7 \%$. Se han documentado metástasis en el área de la glándula parótida, en los ganglios linfáticos cervicales, en el pulmón y en el área mastoidea ${ }^{4,5}$. La lesión suele ser típicamente ulcerada y descamativa, solitaria, de color rosa o blanco, o eritematosa, de aspecto nodular y de 1-2 centímetros.

Se considera un diagnóstico de exclusión basado en la historia clínica y los hallazgos histopatológicos e inmunohistoquímicos. En nuestro caso, la lesión era concordante con un NIC T2 y un FXA en dos superficies oculares distintas (córnea y conjuntiva). Si bien se han reportado otros casos de FXA ocular, para nuestro conocimiento este es el tercer caso reportado de tumores mixtos con un FXA como parte del diagnóstico (Tabla 1).

Los avances en inmunohistoquímica han hecho posible identificar y describir las carácterísticas microscópicas del tumor, y por lo tanto minimizar las posibilidades de diagnósticos erróneos. Histológicamente, el FXA se caracteriza por una combinación de celulas atípicas similares a histiocitos, células fusiformes, células gigantes multinucleadas, algunas de las cuales pueden mostrar vacuolas lipídicas intracitoplasmáticas, y diferentes grados de atipia.
La razón del rápido crecimiento de la lesión puede explicarse por su origen mesenquimatoso fibroblástico, ya que el microambiente tumoral tiene la capacidad de reclutar células mesenquimales con un fenotipo anormal proangiogénico e invasivo ${ }^{6}$.

En el diagnóstico diferencial se incluyen la neoplasia escamosa superficial ocular, el carcinoma epidermoide, el granuloma piógeno, el melanoma amelanótico, el melanoma de células fusiformes pleomórfico, el leiomiosarcoma, el histiocitoma fibroso maligno, el histiocitoma fibroso atípico y el schwannoma maligno ${ }^{7}$.

EI FXA es histológicamente indistinguible de un histiocitoma fibroso maligno, el cual es un tumor que no se asocia a daño actínico y que puede aparecer en cualquier parte del cuerpo. Es más agresivo, tiene mayor posibilidad de presentar metástasis con invasión perineural, linfática y vascular, y su probabilidad de recurrencia a 5 años es del $50 \%$.

El histiocitoma fibroso atípico es el segundo diagnóstico diferencial más común, pudiéndose presentar en niños en áreas no expuestas a daño solar, del tronco y las extremidades. Es un tumor con unas características histológicas y un comportamiento clínico similares a los del FXA ${ }^{7-10}$.

\section{Conclusiones}

EI FXA es una neoplasia cutánea benigna, rara, que se puede presentar en la córnea o la conjuntiva, y debe 
ser considerado como diagnóstico diferencial en tumores de la superficie ocular.

\section{Financiamiento}

Los autores declaran que no recibieron financiamiento.

\section{Conflicto de intereses}

No existe conflicto de intereses por parte de los autores.

\section{Responsabilidades éticas}

Protección de personas y animales. Los autores declaran que para esta investigación no se han realizado experimentos en seres humanos ni en animales.

Confidencialidad de los datos. Los autores declaran que han seguido los protocolos de su centro de trabajo sobre la publicación de datos de pacientes.

Derecho a la privacidad y consentimiento informado. Los autores han obtenido el consentimiento informado de los pacientes y/o sujetos referidos en el artículo. Este documento obra en poder del autor de correspondencia.

\section{Bibliografía}

1. Engelbrecht NE, Ford JG, White WL, Yeatts RP. Combined intraepithelial squamous neoplasia and atypical fibroxanthoma of the cornea and limbus. Am J Ophthalmol. 2000;129:94-6.

2. Kuiper J, Shah A, Kuennen R, Schoenfield L. Atypical fibroxanthoma of the bulbar conjunctiva: a unique case describing the pathology, clinical presentation, and management. Ocul Oncol Pathol. 2018;4:152-6.

3. Shields CL, Shields JA. Tumors of the conjunctiva and cornea. Surv Ophthalmol. 2004;49:3-24

4. Shao L, Newell B, Quintanilla N. Atypical fibroxanthoma and squamous cell carcinoma of the conjunctiva in xeroderma pigmentosum. Pediatr Dev Pathol. 2007:10:149-52.

5. Ullrich K, Wells J, Brennan C, Craig J. Recurrent atypical fibroxanthoma of the limbus. BMJ Case Rep. 2013;2013:bcr2013008762.

6. Galie M, Konstantinidou G, Peroni D, Scambi I, Marchini C, Lisi V, et al Mesenchymal stem cells share molecular signature with mesenchymal tumor cells and favor early tumor growth in syngeneic mice. Oncogene. 2008:27:2542-51.

7. Shieh C, Daluvoy MB, Ellington KS, Proia AD. Atypical fibroxanthoma of the bulbar conjunctiva. Cornea. 2015;34:1620-2.

8. Ly H, Selva D, James CL, Huilgol SC. Superficial malignant fibrous histiocytoma presenting as recurrent atypical fibroxanthoma. Australas $\mathrm{J}$ Dermatol. 2004;45:106-9.

9. Mochizuki K, Yamada T, Mori Y, Sawada A, Mori I, Ohnishi Y. Case of atypical fibroxanthoma in the palpebral conjunctiva. Jpn J Ophthalmol. 2008:52:404-6.

10. Koch M, Freundl AJ, Agaimy A, Kiesewetter F, Kunzel J, Cicha I, et al. Atypical fibroxanthoma - histological diagnosis, immunohistochemical markers and concepts of therapy. Anticancer Res. 2015;35:5717-35. 